\title{
BUILDING A SMART MIRROR FOR THE PURPOSES OF INCREASED Productivity ANd BetTER MENTAL HEALTH, COMPLETE WITH AN APP
}

\author{
Jonathan $\mathrm{Liu}^{1}$ and $\mathrm{Yu} \mathrm{Sun}{ }^{2}$ \\ ${ }^{1}$ Arcadia High School, Arcadia, CA, 91007 \\ ${ }^{2}$ California State Polytechnic University, Pomona, CA, 91768
}

\begin{abstract}
Oftentimes, people find themselves staring in the mirror mindlessly while brushing their teeth or putting on clothes. This time, which may seem unnoticeable at first, can accumulate to a significant amount of time wasted when looked at over the duration of a year, and can easily be repurposed to better suit one's goals. In this paper, we describe the construction and implementation of the Smart Mirror, an intelligent mirror that boasts several features in order to improve an individual's daily productivity. As the name suggests, it is a mirror, and so will not take anything away from the user when he or she is performing their daily teeth brushing. It also hosts facial recognition, and can recognize one's emotions from one glimpse through the camera. The Mirror also comes with an app that is available on the Google Play Store, which helps input tasks and daily reminders that can be viewed on the Smart Mirror UI.
\end{abstract}

\section{KEYWORDS}

Thunkable, Google Firebase, Android, Raspberry Pi

\section{INTRODUCTION}

Especially in the Covid-19 pandemic, but even in general, productivity has decreased for many, including students [1]. This can mainly be attributed to the heightened amount of time spent in front of a screen (whether it be for classes or business meetings) while at home. These computers, while they can be used to do work, also have the ability to play video games or watch forms of entertainment such as YouTube and Netflix. The distractions on laptops are immense, and contribute to decreased productivity because more students are drawn in by the prospect of gaming and less by the possibility of completing homework assignments. After all, which is more fun, video games or analyzing a 17th-century speech? Most students would answer with the former.

In addition, a small but noticeable amount of time is spent in front of the mirror. This time is not utilized to its maximum potential when someone just stares at them self while brushing their teeth. Other actions may be performed, but with the risk of distracting from the original purpose of brushing teeth. This thus presents individuals with a question: how does one increase their efficiency on a day-by-day basis, without being distracted in major ways?

The Smart Mirror aims to solve this issue of decreased productivity. Working as essentially a scaled-up version of a phone interface without apps and just a to-do list, its clean UI allows for

David C. Wyld: CSITY, NWCOM, SIGPRO, ASOFT, AIFZ, BDIoT, ITCCMA, CLSB, DTMN, MLNLP - 2021 pp. 61-71, 2021. CS \& IT - CSCP 2021

DOI: $10.5121 /$ csit.2021.111406 
easy interpretation and usage for people seeking to improve and reach their daily goals. The Android app that accompanies the Mirror further simplifies the purpose of the gadget by providing the local time and other crucial features. The cherry on top is that it is a mirror, so it doesn't distract from everyday tasks such as going out with friends, buying groceries, or driving to work or school. Thus, it is not meant to replace the average Google Calendar or Todoist (a todo list application), but to provide a simple checklist and reminder of tasks that need to be done at the start and end of each day.

There have been very primitive prototypes of a "Magic Mirror" [2] in the past, such as one built by MIT [3] and one by Michael Teeuw [4], but they do not show much besides the basic clock, weather, and news. The user interface is also very messy and clunky, having the news on the left side and the clock directly in the middle. On screens with less than 13 inches of diagonal length, the Magic Mirror is no longer a mirror, but a mash of numbers for the clock and date and words from the news. It is neither aesthetically pleasing nor does it provide effective efficiency for users, as it completely eliminates the original purpose of the device - to still function as a mirror.

A number of social media users and YouTubers have posted videos on building a "Smart Mirror" [5], but they use the same Magic Mirror prototype code mentioned above. No videos seem to have any creators to add their own code to make it more functional and purposeful, and overall, more unique. In addition, in all these videos, the screens and glass used were significantly large, most over 24 inches diagonally. This allows for a better, more aesthetically pleasing interface on larger screens, but does not resolve the fact that the Magic Mirror's UI won't work well on smaller displays.

Our device expands on the "Magic Mirror" [2] mentioned previously, and includes a Raspberry $\mathrm{Pi}$, facial recognition, a to-do list, and Firebase connectivity to the list of features. The input data (for either the to-do list or reminders) gathered from the Android companion app is sent to a Firebase cloud database, which in turn sends these inputs to the Mirror itself. The tasks will then appear in the center-bottom of the Smart Mirror, making for easy visual access while also not distracting from the primary purpose of a regular mirror (to look at oneself). The Mirror also boasts a Raspberry Pi camera, which is used to both detect facial emotions and take a picture of the user if prompted to. Facial recognition is then used to output whatever emotion the algorithm thinks the user is emitting, whether it be joy or regret.

The app that accompanies the Smart Mirror is downloadable in the Google Play Store under the name "Smart Mirror Companion" [6]. It's a simple yet effective app, as it allows individuals to input whatever tasks or reminders they need to complete for the day. And, as mentioned before, this data is sent to Google's Firebase database [7], which the Mirror represents as a section in the center-bottom. Within the app, people can also check off tasks or reminders using the easy switch button. The reminders switch timestamps the input (and users can also edit this to fit their schedule), so people can see when their reminders need to be completed by.

From the list of features described above, it is clear that the Smart Mirror further diversifies and outperforms the skill set of Teeuw's Magic Mirror.

In two experiments described more in detail in Section 4, we demonstrate how the Smart Mirror does, in fact, improve upon daily productivity in the long run. First, we take a look at a six-week period during which users compare the before-and-after of using the Smart Mirror. We analyze their satisfaction with it, and if they found their efficiency increased or decreased. Second, we break down a user who used the Mirror for one month after not using any productivity tools. We analyze her task memory (if she remembered to do tasks better with the Mirror versus not), and also show a graph displaying the results. 
The rest of the paper is structured as follows: Section 2 details the related works which served as the basis of this project; Section 3 focuses on the challenges encountered in the study, and how they were subsequently overcome using preexisting and new methods; Section 4 describes the solutions to these challenges in greater, more intricate detail; Section 5 denotes some experiments we conducted; and Section 6, the last section, will provide a conclusion and offer insight into future work that be done.

\section{RELATED WORK}

The first, most obvious related piece of work would be the Magic Mirror [2, 3, 4]. It served as the basis of the Smart Mirror, and was very friendly in terms of its accessibility and knowledge base. The Magic Mirror documentation already provided the most simple necessities, including time, calendar, and a news feed. But, as pointed out above, it wasn't very user-friendly and certainly was not a mirror for small screens. The Smart Mirror solved this problem using text adjustment, and also includes more features (such as the to-do list and taskbar). It can clearly be seen that the Smart Mirror outclasses the Magic Mirror due to its vast array of features.

Other notable versions of the "Magic Mirror" mostly consist of YouTubers doing DIY projects [5]. Their mirrors, however, utilize the base that is the Magic Mirror mentioned as the first related work, and don't boast any creative or unique aspects and features like the Smart Mirror does.

The YouTubers' DIY videos mostly follow the same script: introduce the concept of a Magic Mirror, download the Magic Mirror documentation found on the Magic Mirror website, purchase and connect the Raspberry Pi, and finally, build the mirror itself using wood, one-sided glass, and glossy finishes [5]. No additional "personality" features were added, so the videos became repetitive and lost their appeal.

\section{Challenges}

In order to build the Smart Mirror, an intelligent mirror that boasts several features to improve an individual's daily productivity, a few challenges have been identified as follows.

\subsection{Challenge 1: Choosing a Method of Displaying the To-Do List}

The first challenge was choosing a method of displaying the to-do list, one of the unique features we decided to implement into the Smart Mirror. After researching several options, we decided on Google's Firebase. It proved to be the most reliable and consistent in terms of displaying list items on the Mirror screen, and was also the simplest to use. However, even with this decision, there was still the issue of connecting to the mirror's program and actually displaying it in the user interface. This meant that our solution needed to contain two steps: one to include a reference to Firebase, and the other to take inputs and data from the database and display it on the screen.

To do this, we implemented Node.js's [12] request function, which asks for an https website link. The parameter was replaced with our Realtime Database link from the Firebase console. This then presented another problem: how could we load the list from the Firebase server, and render it in the Smart Mirror module?

\subsection{Challenge 2: Persistent UI and Aesthetic Issues Requiring Code}

The second challenge after implementing Firebase presented itself when we tried to run the program. The primary device at the time was a MacBook Air, and when our edited Magic Mirror 
ran on Electron [8], the interface was found to be somewhat clunky and could not be personalized (i.e., specific panels could not be added or deleted; see Figure 1). The obvious solution was to switch to a larger screen, but the original problem still persisted (users with smaller screens would lose the "mirror" aspect of the device and instead just view text). We decided to utilize proportions to attempt to fix this issue, and this meant quite a few lines of code to fix the text adjustment and tile sizes.

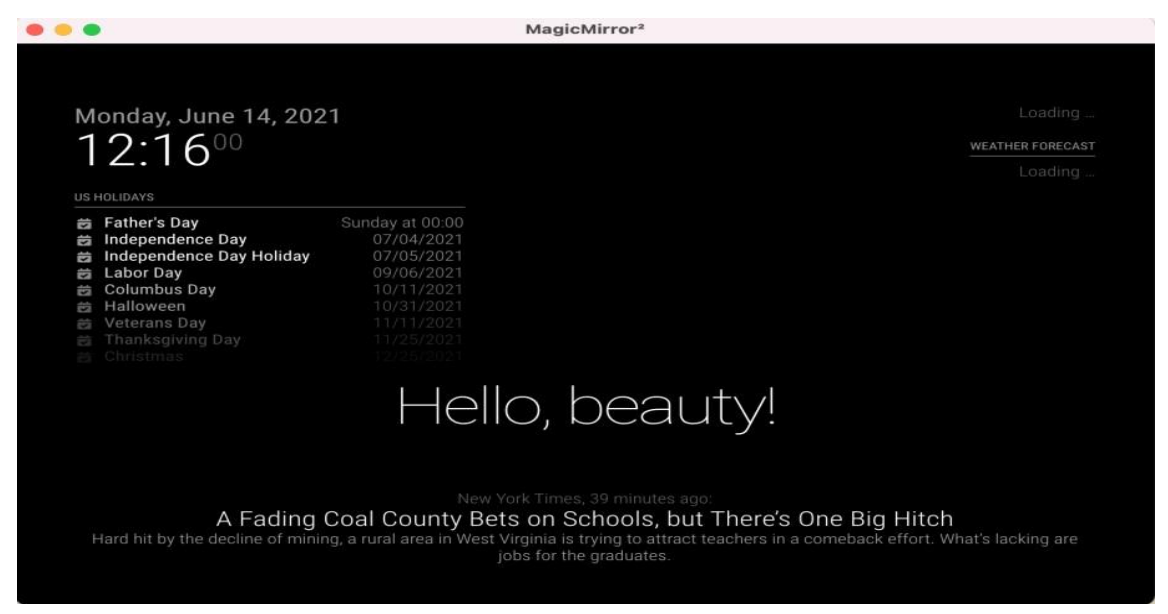

Figure 1. Magic Mirror display

The original Magic Mirror display is shown in Figure 1. As can be seen, there is no longer the "mirror" aspect with a small display.

\subsection{Challenge 3: Logistic Challenges of Building the Mirror}

The third challenge was building the mirror itself. This proved to be harder than initially thought, as it required detailed planning and organization of where each component was to go. Parts such as the Raspberry Pi and its accompanying camera, along with its wires, needed to be placed in spots where it wouldn't make the mirror seem too bulky or thick, for example. One-sided glass was also in limited supply because of the pandemic. In addition, the Raspberry Pi was somewhat loud when the Smart Mirror program ran, so we had to include additional cooling features in order to lower the sound (because no one wants a loud fan whirring behind a mirror).

\section{Our Proposed Solution}

First, we implemented the code found in our research for a Smart Mirror, which can be found on the Magic Mirror website. This provided us with all the basic necessities, such as the clock and news panels (the portions of the mirror that were already visible when the program was run). After a quick scan of the folders included within the Magic Mirror program, we deduced that the "modules" folder would be our main focus, as it was the folder that contained all the "customizing" that needed to be done (including the camera, to-do list implementation, etc.)

Second, Google Firebase needed to be integrated into the program. We chose Firebase because it was the simplest and relatively easy to understand. Backend data could also be easily viewed by a few clicks, so not much effort is required (see Figure 2). 


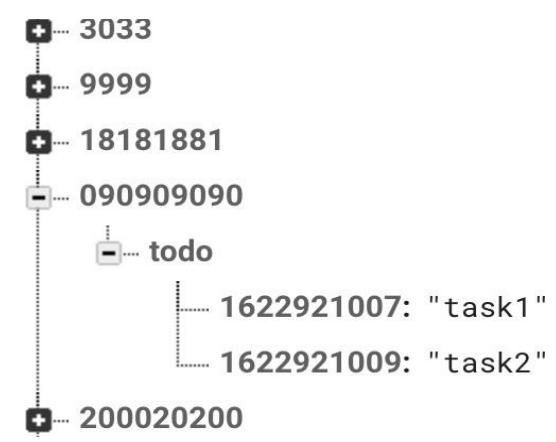

Figure 2. A screenshot of the Realtime Database in Firebase. The numbers represent test device ID's, and the text under "todo" represent the inputs entered by the user.

We implemented this and the Firebase into our code, as shown in Figure 3. The broadcastTodoList() function displays the To-do List found in the Firebase Realtime Database.

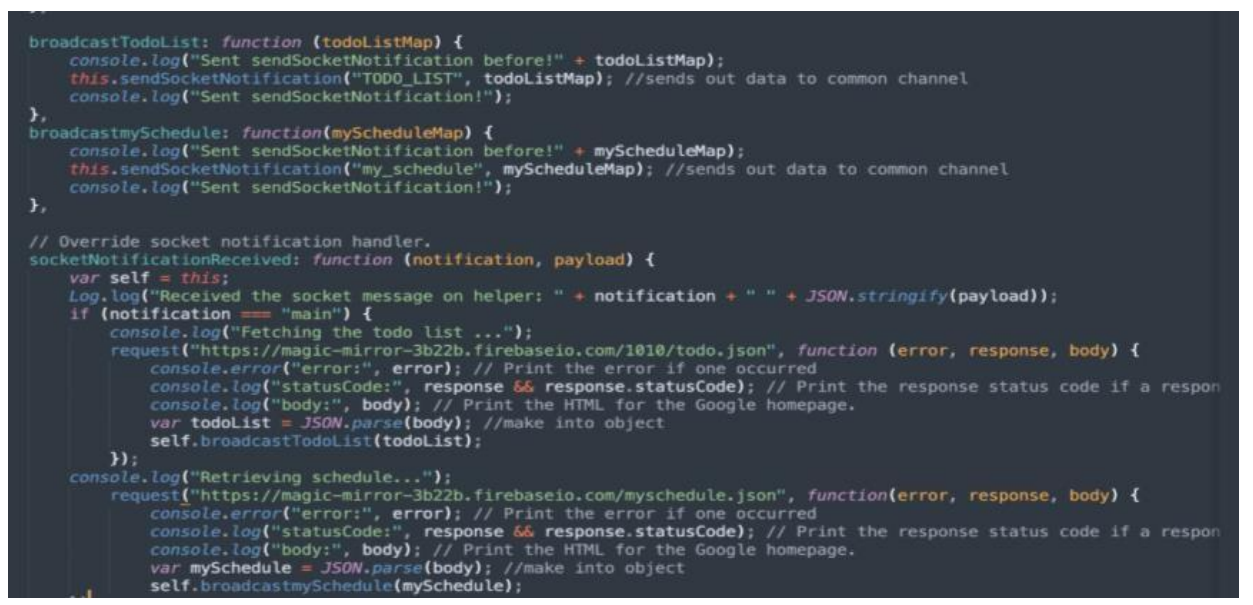

Figure 3. A snippet of the added code.

The next step was to include the camera feature, as it was an integral part of the creative and unique aspect of the Smart Mirror. To do this, we considered whether to create our own facial recognition program, or just find a self-sustaining program on GitHub [9] and paste that into the mirror's program. After some research, we decided on the latter because it would save a significant amount of time that we could use to improve other aspects of the Smart Mirror. Some detailed facial recognition examples were found on GitHub, but we settled on a seemingly complicated, yet simple program because it boasted the ability to detect emotion and print it onto the screen [11]. It also wasn't large in file size, which was important due to the Raspberry Pi and its loud fans. From there, it fit right in with the "modules" folder in the Smart Mirror, and so allowed for easy access if any revisiting of the code was needed (see Figure 4). 


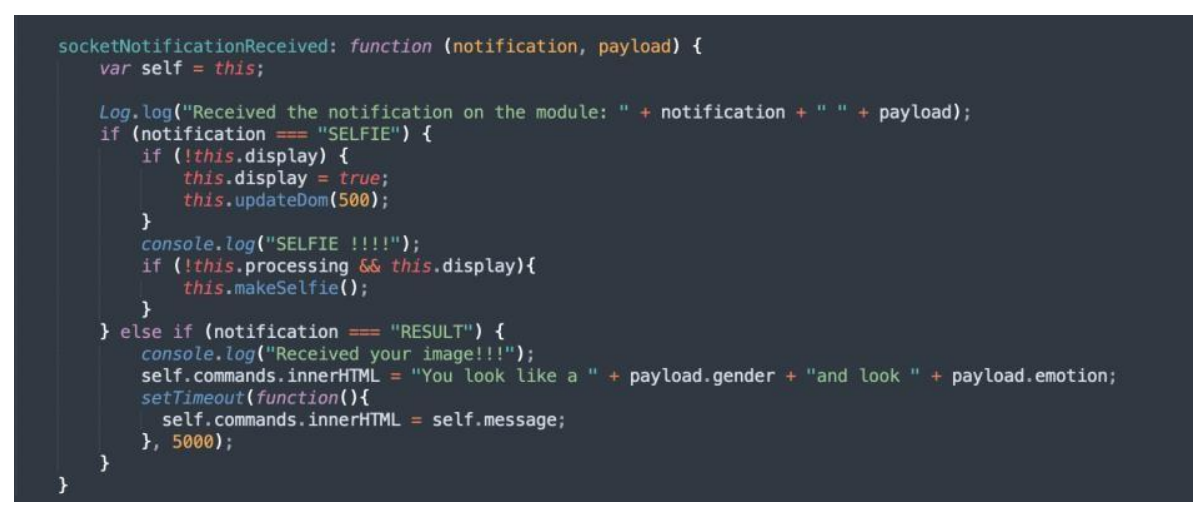

Figure 4. A portion of the camera program that prints out the user's gender and / or facial emotions after taking a selfie.

Next was the usage of a Raspberry Pi to be able to run the program. Raspberry Pi was recommended by the Magic Mirror creators, and it's also small enough to not be any large hindrance in terms of size or width of the actual mirror itself. A Raspberry Pi Camera is also used since it pairs with the Raspberry $\mathrm{Pi}$ and does not require extra steps to connect to the $\mathrm{Pi}$. We followed the steps on the Raspberry Pi website [10] to build it. The three heatsinks that were included in the Raspberry Pi package were placed onto the chips so that the fan didn't have to work extremely hard (and thus produce overly loud sound) to cool the device.

While the Smart Mirror is not touchscreen, it can be controlled via a mouse if the user needs to. However, much of the actions can be done through the Smart Mirror Companion App, which allows users to input their daily tasks and reminders. These list items will then be sent to the Mirror and will show in the user interface. As such, there is really no need for a mouse.
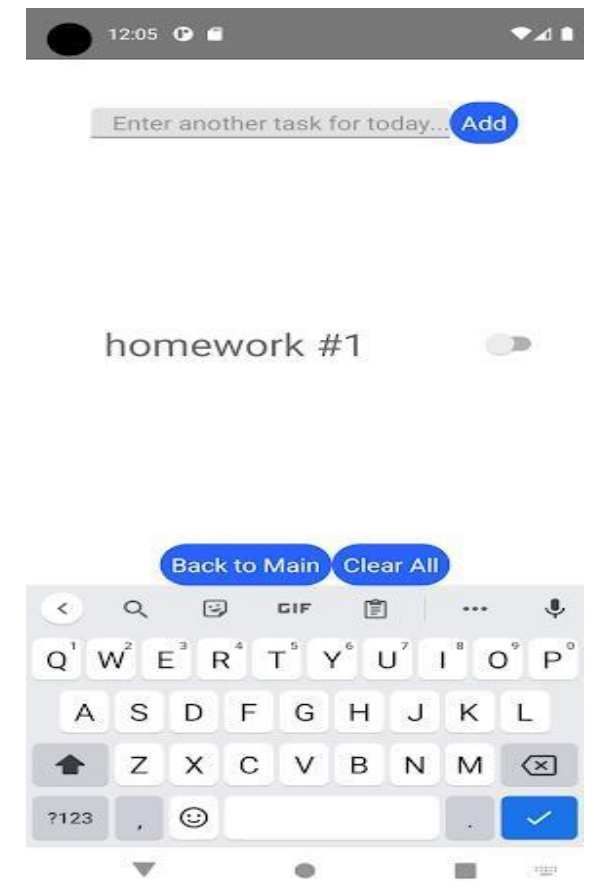

Figure 5. Screenshot of the App, which can be found on the Google Play Store; "Enter text" box allows users to type in their tasks, which will be displayed in the Mirror interface. 


\section{EXPERIMENTS}

\subsection{Experiment 1}

To provide a quantifiable experiment to prove improvement with the Smart Mirror, we first asked around two hundred people to see if they would like to participate. About 53 responded. In this experiment, we wanted to see if the Mirror really lived up to the above claims of increased efficiency. Over a three-week period, participants were surveyed weekly on how efficiently they were able to complete or simply just remember tasks. Then, in another three-week period, the same people used the Smart Mirror and continued to perform their daily routines. After the entire experiment was over, another survey was sent out, asking the people if they noticed that their memory improved or were able to complete tasks earlier on in the day. 46 people, or about $87 \%$, individuals out of the 53 noted that they were able to both remember and finish tasks off more easily when using the Smart Mirror (see Figure 6).

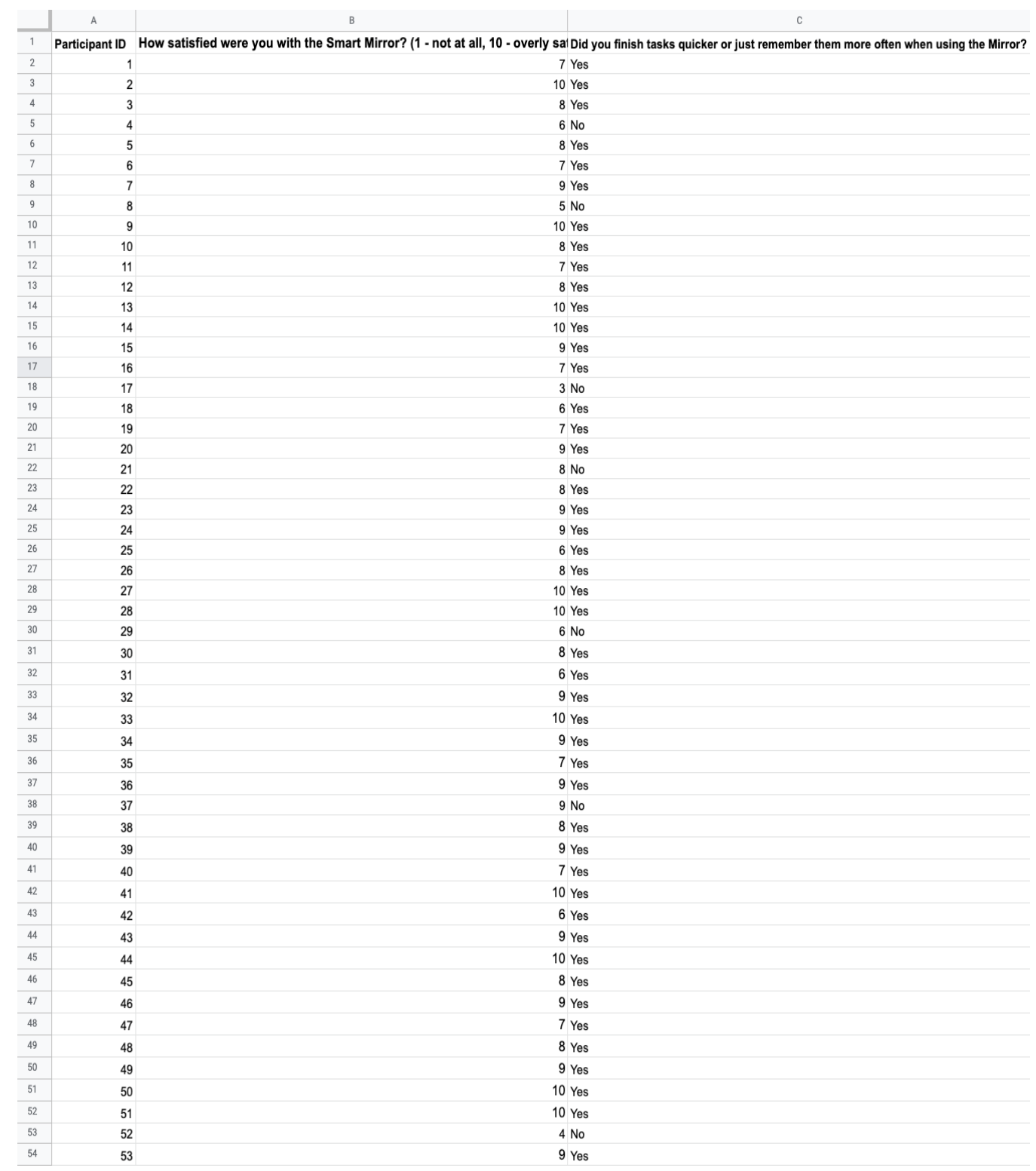

Figure 6. Satisfaction with the Smart Mirror ranked on a scale of 1-10 for 50(+) participants. 
The first question was merely a survey question that allowed us to access feedback based on 53 users. Many scored the Mirror above 5 (a good sign, of course), with some finding it less than ideal and not living up to expectations. The average value of these participants' scores was 8.07, a high score for such an early version of a product. In addition, in a separate "feedback" section, users told us what they would have liked to see after using the Smart Mirror. This will be discussed in the last paragraph of this subsection.

From the table data provided, it is quite clear that while many users found the Smart Mirror unsatisfactory, participants still were able to finish tasks quicker, or, more generally, remember them when using the Mirror. Only 7 people responded "no" to the second question, which is a small minority of the total participants. However, these seven individuals all did not rate the Mirror "badly" (i.e. scores of 5 or less); some gave the Mirror a relatively high score. It can be inferred that the Mirror itself was useful and interesting, but the "productivity" aspect was not as beneficial to them.

The fact that many users thought that the Mirror was unsatisfactory can most probably be attributed to the still-clunky user interface. While our version improves on the interface provided by the Magic Mirror (it introduces text adjustment, for example), it still does not allow people to hide or add additional panels. For instance, if one wanted to integrate their own calendar and sync it with Google Calendar, the Smart Mirror cannot do that. As such, it is understandable why many submissions put scores of 5 or lower as the answer to the first question ("How satisfied were you with the Smart Mirror? (1 - not at all, 10 - overly satisfied)"). This feature of adding/eliminating panels is part of our future work on the Smart Mirror (further information in Section 6).

\subsection{Experiment 2}

Our second experiment involved only one person, but lasted two months. The idea was to have one person record the number of tasks she forgot within a month (each day counting how many tasks she forgot, for 31 days). Then, in the second month, the subject used the Smart Mirror and continued to $\log$ the number of forgotten tasks. Each number was recorded in a Google Spreadsheet (see Figure 7). 


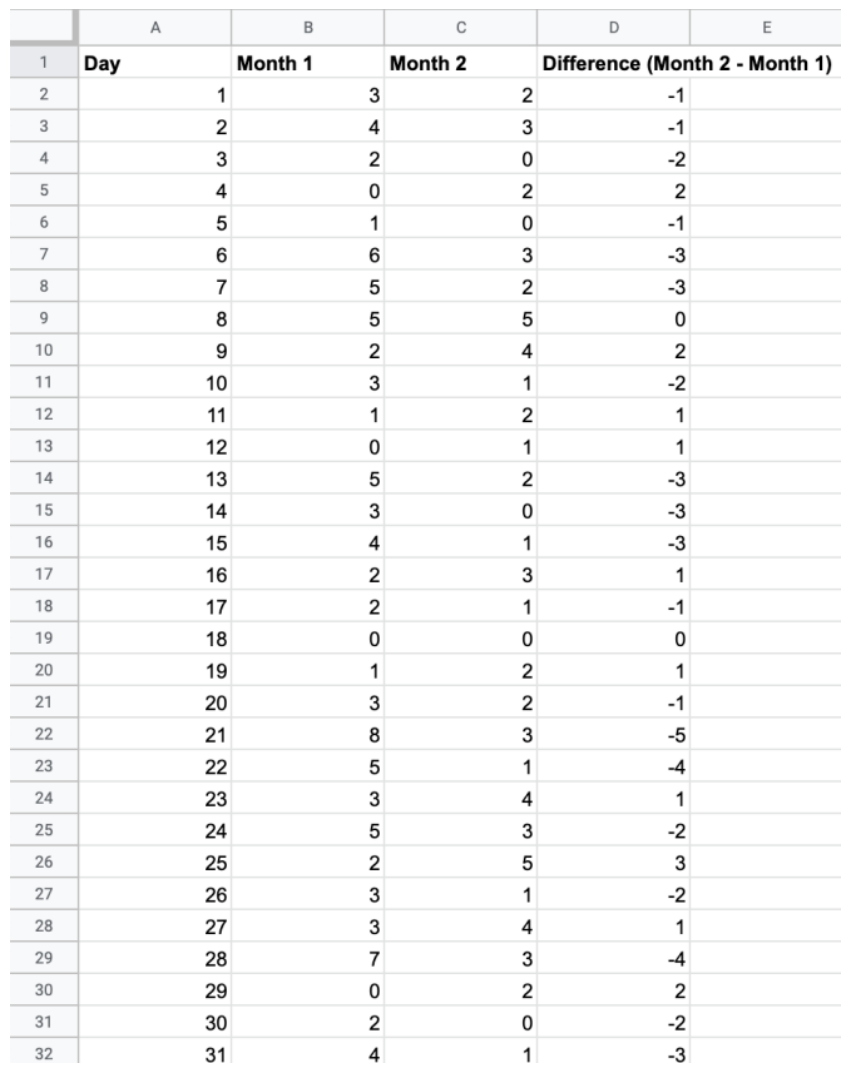

Figure 7. Results of Experiment 2; forgotten tasks without Smart Mirror (month one) and with Smart Mirror (month two)

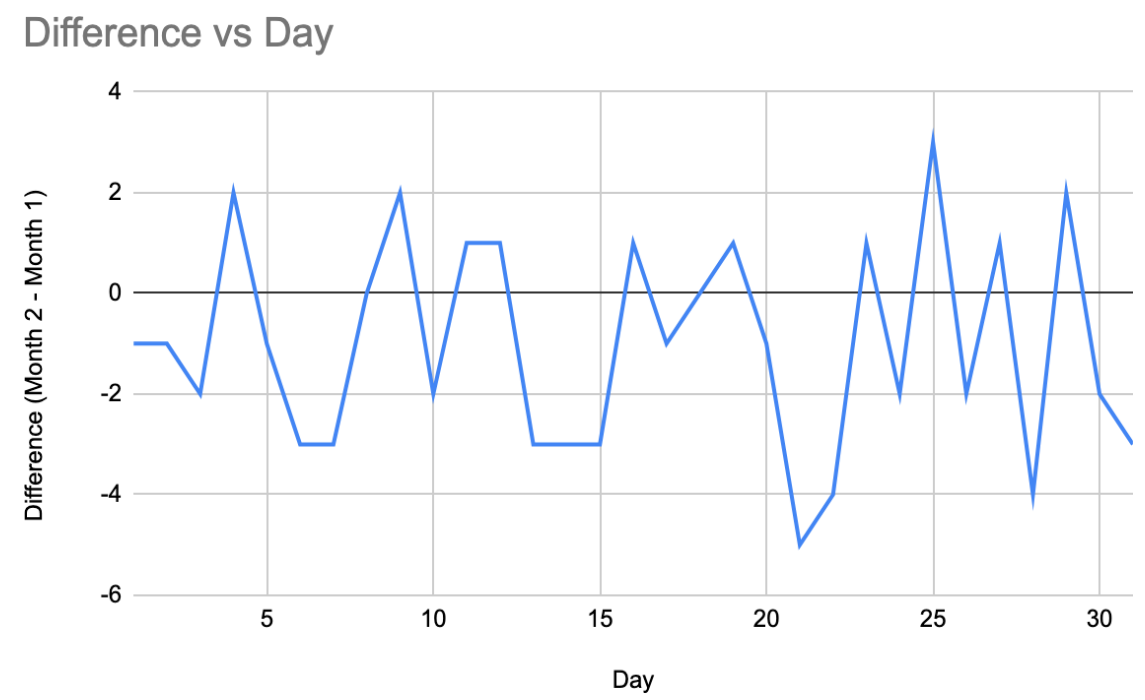

Figure 8. The graph of the data collected in Experiment 2.

As seen in the graph, much of the data points lie below the 0 line, meaning more tasks weren't forgotten when using the Smart Mirror. Thus, it can be claimed that the Smart Mirror improves productivity, as the person remembered to do more tasks when using the Mirror. We also provided a feedback form to the participant to see if there was anything we could improve on. Her only suggestion was for us to include more customizable options, as she didn't really need the Holidays panel. This is the common denominator in the previous experiment: people want the 
Mirror to be customizable and to be able to add or remove certain parts of the Mirror. And, as mentioned, this will be discussed in Section 6, "Future Works."

These two experiments were aimed to prove the idea that the Smart Mirror improves productivity. And, judging from the results of both experiments, it is quite clear that the Mirror does what we intended it to do. It not only allows people to finish tasks quicker (results of Experiment 1), it also just simply lets users remember them more easily (results of Experiment 2). Thus, it reaches our expectations, but just barely. There are still a number of more things to work on, such as allowing users to add or remove panels.

\section{CONClusion AND Future Work}

The Smart Mirror is a new and improved version of the Magic Mirror built by inventor Michael Teeuw [4]. Initially conceived to solve the problem of decreased productivity (due to the effects of the pandemic), it grew into a personal project where we wanted to customize the mirror and allow others besides ourselves to enjoy it. As such, it possesses more features, including a to-do task bar and calendar. It adds to the list of features found with the Magic Mirror (time, news feed, major holidays $[15,16])$. In order to include our desired additional features, we needed to connect Google's Firebase and design an additional app, which is called the "Smart Mirror Companion App" [6], which can be found on the Google Play Store. Automatic text adjustment was also added so that smaller screens could enjoy the Smart Mirror and not be hindered by large amounts of text.

The most significant limitation was the building of the mirror. It required a frame (wood or metal), a Raspberry Pi and accompanying camera, and one-sided glass. On top of that, it also required some building or craftsmanship skills. After all, a list of materials is only a list; there is still the issue of building the mirror itself, and making sure nothing goes wrong during the process. For people who are not skilled with their hands or have no experience in building things, this would be a tedious procedure.

In the near and distant futures, more thought will be directed towards optimizing the build quality of the mirror (such as the optimal placement of the Raspberry Pi) while still allowing unskilled persons to successfully build their own Smart Mirror. If the Smart Mirror were ever to be marketed and sold, this issue would definitely need to be solved beforehand so as to provide the most customer satisfaction.

In addition to these additions, more unique features can be added upon request. One such feature that we are already thinking about adding is connecting Google Calendar [13] or Todoist [14] to the Mirror. This would completely eliminate the need for our additional app, as it can just display events or tasks that users can input easily via their computer.

\section{AUTHORS' NOTES}

Although many reviewers have requested the GitHub for the Smart Mirror (and we respect their thoughts and inputs), we would like to keep it private in case the Mirror becomes a marketable product in the future. 


\section{REFERENCES}

[1] "Gendered Effects of Covid-19 on Scientific Productivity" https://journals.sagepub.com/doi/full/10.1177/23780231211006977

[2] MagicMirror - https://magicmirror.builders/

[3] MIT Magic Mirror - https://courses.media.mit.edu/2016spring/mass65/2016/05/14/the-magic-mirror/

[4] Inventor of the MagicMirror, Michael Teeuw - https://michaelteeuw.nl/

[5] Example of YouTube "DIY Smart Mirror" - https://youtu.be/OYlloiaBINo

[6] "Smart Mirror Companion App", Google Play https://play.google.com/store/apps/details?id=com.gmail.jj2004liu.magicmirrorv1

[7] Google Firebase - https://firebase.google.com/

[8] Electron - https://www.electronjs.org/

[9] Facial Recognition Programs on Github - https://github.com.cnpmjs.org/topics/face-recognition

[10] Raspberry Pi Camera Usage - https://raspberrytips.com/install-camera-raspberry-pi/

[11] Github, MagicMirror Camera Module - https://github.com/alexyak/camera

[12] Node.js - https://nodejs.org/en/

[13] Google Calendar - https://calendar.google.com/

[14] Todoist, one of the most popular to-do checklist apps - https://todoist.com//

[15] Newsfeed - https://rss.nytimes.com/services/xml/rss/nyt/HomePage.xml

[16] Major Holidays - https://www.timeanddate.com/holidays/us/

(C) 2021 By AIRCC Publishing Corporation. This article is published under the Creative Commons Attribution (CC BY) license. 